\title{
Design of a Microwave Duplexer without Ferrite and without Magnet
}

\author{
Fethi MEJRI, Taoufik AGUILI
}

Sys'Com Research Laboratory, National Engineering School of Tunis (ENIT), University Tunis El Manar, Tunis, Tunisia

fethi_mejri@laposte.net, taoufik.aguili@gmail.com

Submitted August 6, 2017 / Accepted November 22, 2017

\begin{abstract}
In this paper we present the design, realization and characterization of a microwave duplexer, compact, easy to realize and integrate into systems such as ground penetrating radars. It is made without the use of ferrite or magnet. This device is designed in the $S$ band and made in micro-ribbon technology. It consists of a power divider and two RF amplifiers, low gain, using a BFR91 bipolar transistor. The latter is frequently available and inexpensive. Measurements made on a vector network analyzer have shown a low insertion loss with insulation considered satisfactory - for low power applications - between the transmitter (Tx) and the receiver (Rx) circuits.
\end{abstract}

\section{Keywords}

Duplexer, micro-strip, microwave, bipolar transistor, amplifier, polarization tee, Wilkinson power divider

\section{Introduction}

A duplexer (Fig. 1) is an electronic device allowing the use of the same antenna for transmitting and receiving the signal [1], while respecting the optimal adaptation of the antenna for each input. It comprises a transmission channel (Tx), a reception channel $(\mathrm{Rx})$ and an antenna channel $(\mathrm{Tx} / \mathrm{Rx})$. The transmission and reception channels (Tx and Rx) are completely isolated. It is therefore a switch that connects the transmitter to the antenna and then the antenna to the radio receiver. It must prevent the pulses of considerable power transmitted by the transmitter from damaging or destroying the circuits of the receiver, which are calibrated for the processing of signals of very low power. Generally a duplexer is used in radar systems and in wireless communications systems. There are different types of duplexers that operate on different principles, including:

- Hybrid circle duplexer (ferrite and magnet based).

- Coaxial resonant cavity or waveguide.

-with TR (Transmission-Receiving) and ATR (AntiTransmission-Receiving) tubes;

- or with PIN diodes.

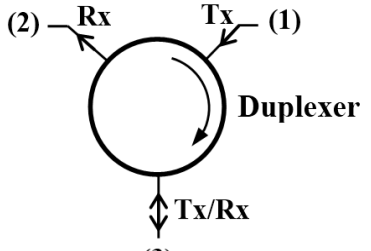

(3)

Fig. 1. Synoptic diagram of the duplexer.

The primary function of TR tubes is to direct the return echoes to the receiver and to protect it from the transmitted pulse [2-5]. They are usually spark gaps in a partially vacuum tube. An electrical arc can be generated in the tube by the ionization of the gas it contains by a sufficient voltage between the electrodes.

The primary function of the ATR tubes is to cut the channel to the transmitter for any pulse moving in the transmission line [4], [5]. They are in fact simplified TR tubes. They are filled with an inert gas like argon because the recovery time is not critical. In addition, an exciting agent, such as a maintenance electrode, is not required. The fact of not having an active gas and a maintenance electrode makes it possible to obtain a tube having a longer life than the tubes TR.

Figure 1 shows the block diagram of a duplexer. Its matrix $\boldsymbol{S}$ is defined below:

$$
[\boldsymbol{S}]=\left[\begin{array}{lll}
S_{11} & S_{12} & S_{13} \\
S_{21} & S_{22} & S_{23} \\
S_{31} & S_{32} & S_{33}
\end{array}\right]=\left[\begin{array}{lll}
0 & 0 & 0 \\
0 & 0 & 1 \\
1 & 0 & 0
\end{array}\right] .
$$

The problem is that these types of devices sometimes exist only among sellers of radio frequency components, which exist in their turns only in developed countries. Even to acquire these, it takes time to deliver. Moreover, these devices do not exist for all frequency ranges. This problem prompted us to design and build a duplexer based on an element that can exist in all sellers of electronic components. This element is the bipolar transistor BFR91 of the NPN type. It has a cut-off frequency equal to $6 \mathrm{GHz}$. It can dissipate power up to $300 \mathrm{~mW}$. It has input and output impedances equal to $50 \Omega$. Its theoretical gain, of maximum power transfer, is equal to $5 \mathrm{~dB}$ at $2.4 \mathrm{GHz}$. The 


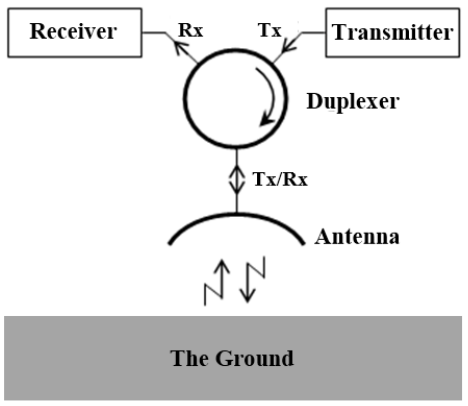

Fig. 2. Synoptic diagram of a GPR radar.

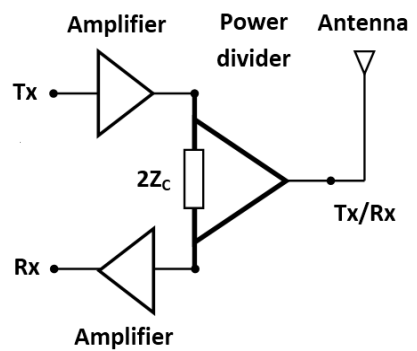

Fig. 3. Synoptic diagram of the studied duplexer.

duplexer realized with the help of this transistor presents multiple advantages such as low manufacturing cost, simplicity of design and ease of reproducibility. This device will subsequently be used in a GPR RADAR [6], [7] application operating at $2.4 \mathrm{GHz}$ (Fig. 2).

\section{Description of the Duplexer}

The duplexer is realized in planar technology and its working frequency is equal to $2.4 \mathrm{GHz}$. It is composed of two RF amplifiers, based on the bipolar transistor BFR91, and a Wilkinson power divider. The transistors used are of the NPN type and are of low power.

This device is realized on a printed circuit that is frequently available and inexpensive. The conductor constituting it is copper, with a thickness $T=35 \mu \mathrm{m}$ and a conductivity $\sigma=59.6 \times 10^{6} \mathrm{~S} / \mathrm{m}$. Its dielectric is Teflon glass, with a thickness $H=1.5 \mathrm{~mm}$, with a relative permittivity $\varepsilon_{\mathrm{r}}=4.32$ and having losses modeled by $\tan \delta=0.02$. The synoptic diagram of this duplexer is shown in Fig. 3.

\subsection{Characterization of the RF Amplifier}

The amplifier used is shown in Fig. 4. It is based on a bipolar transistor BFR91 which is mounted as a common emitter. The impedances of the input and the output of this transistor are equal to $50 \Omega$. To ensure impedance matching for maximum power transfer, the input and output of the transistor have been connected to microstrip lines with characteristic impedance $Z_{\mathrm{C}}=50 \Omega$. The latters have a track width equal to $2.86 \mathrm{~mm}$. It is calculated by the ADS LineCalc tool. The polarization of the transistor takes place via a low-pass filter LC circuit which blocks the passage of the RF signal to the supply so that it is not disturbed and

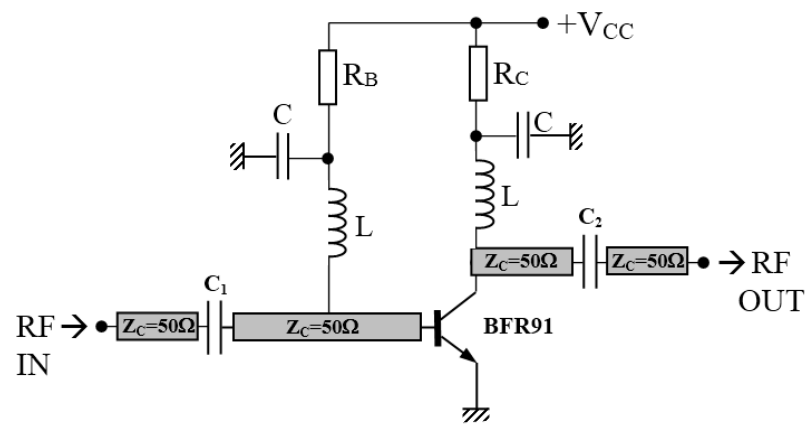

Fig. 4. Electrical diagram of the realized $\mathrm{RF}$ amplifier $\left(V_{\mathrm{CC}}=+12 \mathrm{~V}, \quad R_{\mathrm{B}}=33 \mathrm{k} \Omega, \quad R_{\mathrm{C}}=220 \Omega, \quad C_{1}=C_{2}=\right.$ $10.4 \mathrm{nF})$.

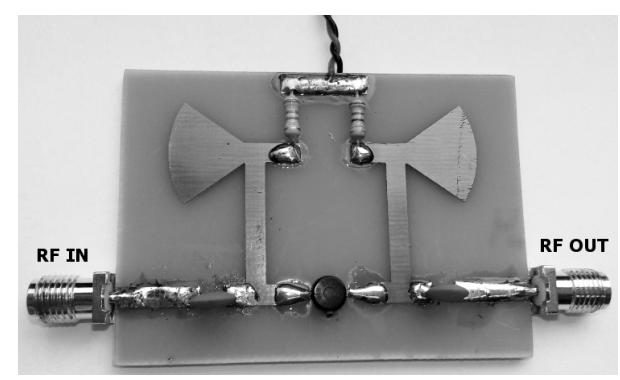

Fig. 5. Topology and design of the RF amplifier.

\begin{tabular}{|c|c|c|}
\hline [S] Parameters & Module [dB] & Phase [deg] \\
\hline$S_{11}$ & -13.4 & 84 \\
\hline$S_{21}$ & 3.4 & 56 \\
\hline$S_{12}$ & -12.7 & 83 \\
\hline$S_{22}$ & -12.52 & -90 \\
\hline
\end{tabular}

Tab. 1. Results of measurements of parameters $S$ of the active circuit.

allows the direct current of the power supply to pass to the transistor which is polarized. The rest point of the transistor is set by $R_{\mathrm{B}}$ and $R_{\mathrm{C}}, I_{\mathrm{C} 0} \approx 20 \mathrm{~mA}$. The impedances of the connection capacitors $C_{1}=C_{2}=10.4 \mathrm{nF}$ are chosen to be strictly less than $50 \Omega$ at the working frequency $f=2.4 \mathrm{GHz}$.

\section{- Stability and Gain:}

Table 1 represents the $S_{i j}$ parameters of the RF amplifier of Fig. 5. These parameters $S_{i j}$ are obtained by measurements on a vector network analyzer (VNA) at the frequency $f=2.4 \mathrm{GHz}$.

These parameters $\mathrm{S}$ make it possible to calculate the Rollet factor $K$ which intervenes in the stability criterion [8]

$$
K=\frac{1+|\Delta|^{2}-\left|S_{11}\right|^{2}-\left|S_{22}\right|^{2}}{2\left|S_{12} S_{21}\right|}
$$

with

$$
|\Delta|=S_{11} S_{22}-S_{21} S_{12} \text {. }
$$

Since $K=4.294>1$ and $|\Delta|=0.119<1$, we can deduce that the active circuit will be unconditionally stable at $2.4 \mathrm{GHz}$. The determination of the Rollet factor $K$ is necessary to obtain also the maximum gain at $2.4 \mathrm{GHz}$. When 
the amplifier is adapted to input and output, its maximum theoretical gain is given by:

$$
G_{\max }=\frac{\left|S_{21}\right|}{\left|S_{12}\right|}\left(K-\sqrt{\left(K^{2}-1\right)}\right)=6.82 \mathrm{~dB} .
$$

In practice this theoretical gain is not really accessible.

\section{- Input and output impedances:}

They are provided from the value of the reflection coefficients $\Gamma_{\mathrm{e}}$ and $\Gamma_{\mathrm{s}}$ by the relation [8]:

$$
z=\frac{1+\Gamma}{1-\Gamma}
$$

Input adaptation:

$$
\Gamma_{\mathrm{e}}=C_{1}^{*}\left(\frac{B_{1}-\sqrt{B_{1}^{2}-4 \cdot\left|C_{1}\right|^{2}}}{2 \cdot\left|C_{1}\right|^{2}}\right)
$$

with:

$$
\begin{aligned}
& B_{1}=1+\left|S_{11}\right|^{2}-\left|S_{22}\right|^{2}-|\Delta|^{2}, \\
& C_{1}=S_{11}-\Delta S_{22}^{*} .
\end{aligned}
$$

We find a coefficient of reflection:

$$
\Gamma_{\mathrm{e}}=4.55 \times 10^{-4}-\mathrm{j} 4.1 \times 10^{-2}
$$

which gives $Z_{\mathrm{IN}}=49.88-\mathrm{j} 4.10$.

Output adaptation:

$$
\Gamma_{\mathrm{s}}=C_{2}^{*}\left(\frac{B_{2}-\sqrt{B_{2}^{2}-4 \cdot\left|C_{2}\right|^{2}}}{2 \cdot\left|C_{2}\right|^{2}}\right)
$$

with:

$$
\begin{aligned}
& B_{2}=1+\left|S_{22}\right|^{2}-\left|S_{11}\right|^{2}-|\Delta|^{2}, \\
& C_{2}=S_{22}-\Delta S_{11}^{*} .
\end{aligned}
$$

We find a coefficient of reflection:

$$
\Gamma_{\mathrm{s}}=3.13 \times 10^{-3}+\mathrm{j} 5.22 \times 10^{-2}
$$

which gives: $\quad Z_{\text {OUT }}=50.04+\mathrm{j} 5.25$.

The impedances of the input and the output of the amplifier are almost equal to $50 \Omega$, which means that they do not require any adaptation device.

\section{- Insulation circuit}

The polarization of the BFR91 transistor takes place via a broadband isolation circuit $(B P=1 \mathrm{GHz}$, see Fig. 7), realized in micro-ribbon technology. It is a low pass filter that allows the direct current of the power supply to pass to the transistor and blocks the passage of the RF signal to the power supply so that it is not disturbed. The latter consists of distributed elements, a series inductance and a parallel capacitor (Fig. 6).

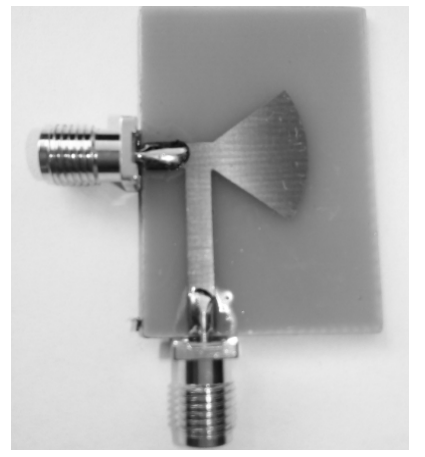

Fig. 6. Topology and design of the insulation circuit.

The shape of the butterfly capacitor [9], [10] allows it to operate in a wide frequency band. It also makes it possible to reduce its radiation with respect to a capacitor of square or rectangular shape. Its length is chosen equal to $\lambda_{\mathrm{g}} / 4$ and is obtained only by optimization under the ADS software since its width is increasing so its characteristic impedance and its effective permittivity vary according to its length.

$$
\lambda_{\mathrm{g}}=\frac{\lambda_{0}}{\sqrt{\varepsilon_{\mathrm{eff}}}}=\frac{c}{f \cdot \sqrt{\varepsilon_{\mathrm{eff}}}} .
$$

The butterfly capacitor is a planar line which obeys the relation (11). Its length is equal to $\lambda_{\mathrm{g}} / 4$ and it is open at its end. It returns to its input a short circuit for the RF signal [11].

$$
Z(x)=Z_{\mathrm{c}} \frac{Z_{\mathrm{ch}}+\mathrm{j} Z_{\mathrm{c}} \tan (\beta x)}{Z_{\mathrm{c}}+\mathrm{j} Z_{\mathrm{ch}} \tan (\beta x)} \Rightarrow Z\left(x=\frac{\lambda}{4}\right)=0 .
$$

The short circuit brought back to the input of the capacitor short circuits the output of a line of $\lambda_{\mathrm{g}} / 4$ length. This line brings back to its input an open circuit for the RF signal.

$$
Z\left(x=\frac{\lambda}{4}\right)=\infty \text {. }
$$

Figure 7 shows the S parameters obtained by simulations on ADS software and by measurements made by a VNA - of the isolation circuit of Fig. 6. Figure 7 shows that for a direct current the reflections $\left(S_{11} \approx S_{22}\right)$ are negligible, of the order of $-70 \mathrm{~dB}$, and the transmissions $\left(S_{21} \approx S_{12}\right)$ are maximum, of the order of $0 \mathrm{~dB}$. On the other hand, for the RF signal at $2.4 \mathrm{GHz}$, the reflections are maximum, equal to $-0.53 \mathrm{~dB}$, and the transmissions are negligible, equal to $-28.8 \mathrm{~dB}$.

In conclusion, the isolation circuit behaves as an open circuit for the RF signal and as a direct current wire. Thus, this circuit is used to block the RF signal and let the direct current pass through (low pass filter).

We also note from Fig. 7 that the bandwidth of this isolation circuit is equal to $1 \mathrm{GHz}$. On this band the reflection coefficients are strictly superior to $-3 \mathrm{~dB}$ and the transmission coefficients are strictly lower than $-10 \mathrm{~dB}$. We can also say that the bandwidth of the RF amplifier is linked to this isolation circuit. 


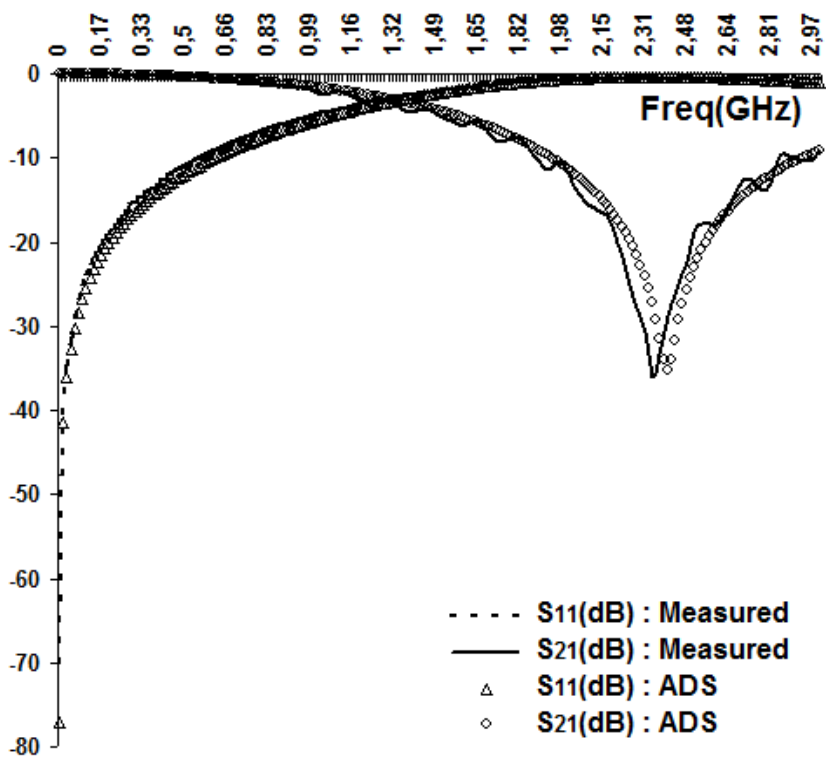

Fig. 7. $S_{i j}$ parameters of the isolation circuit.

\subsection{Characterization of the Wilkinson Power Divider}

Figure 8 shows Wilkinson's power divider. The Wilkinson divider [11-14] is composed of two quarter wave lines and a resistance $R=2 Z_{\mathrm{c}}=100 \Omega$ at the end of these two lines. The characteristic impedance of the supply lines is equal to $Z_{\mathrm{c}}=50 \Omega$. The two quarter-wave lines have a characteristic impedance $Z_{\mathrm{c}}^{\prime}$ such as:

$$
Z_{\mathrm{c}}^{\prime}=Z_{\mathrm{c}} \sqrt{2}=70.71 \Omega .
$$

$Z_{\mathrm{c}}=50 \Omega$ corresponds to a track width equal to $2.86 \mathrm{~mm}$. $Z_{\mathrm{c}}^{\prime}=70.71 \Omega$ corresponds to a track width equal to $1.49 \mathrm{~mm}$. These track widths are calculated by the ADS LineCalc tool.

All inputs of the Wilkinson power divider are adapted, the outputs are symmetrical and isolated from each other. The energy entering through the port 1 will be divided into two equal parts. The first half will be directed to port 2 and the second half to port 3. The two-fold division of energy is not an obligation. It may be unevenly distributed, but less frequently than an equi-distribution.

Figure 9 shows the power divider which is realized. Its matrix $\mathrm{S}$ is defined below:

$$
[S]=\left[\begin{array}{lll}
S_{11} & S_{12} & S_{13} \\
S_{21} & S_{22} & S_{23} \\
S_{31} & S_{32} & S_{33}
\end{array}\right]=\left[\begin{array}{ccc}
0 & \frac{-\mathrm{j}}{\sqrt{2}} & \frac{-\mathrm{j}}{\sqrt{2}} \\
\frac{-\mathrm{j}}{\sqrt{2}} & 0 & 0 \\
\frac{-\mathrm{j}}{\sqrt{2}} & 0 & 0
\end{array}\right] .
$$

Figure 10 shows $S_{11}=-23.6 \mathrm{~dB}$ and $S_{22}=S_{33}=$ $-24.2 \mathrm{~dB}$, for $f=2.4 \mathrm{GHz}$. This means that the three ports of the power divider are well adapted.

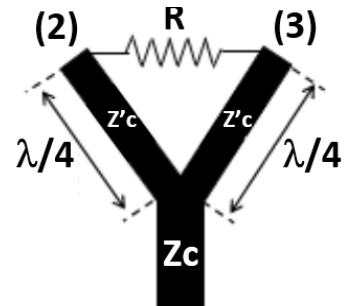

(1)

Fig. 8. Wilkinson power divider.

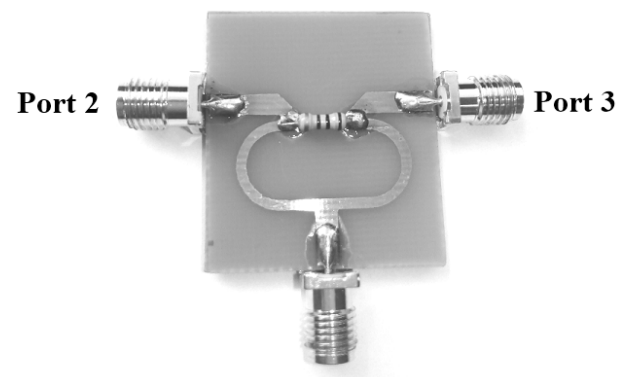

Port 1

Fig. 9. The achieved Wilkinson power divider.

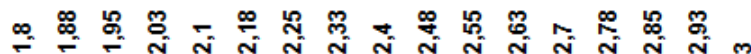

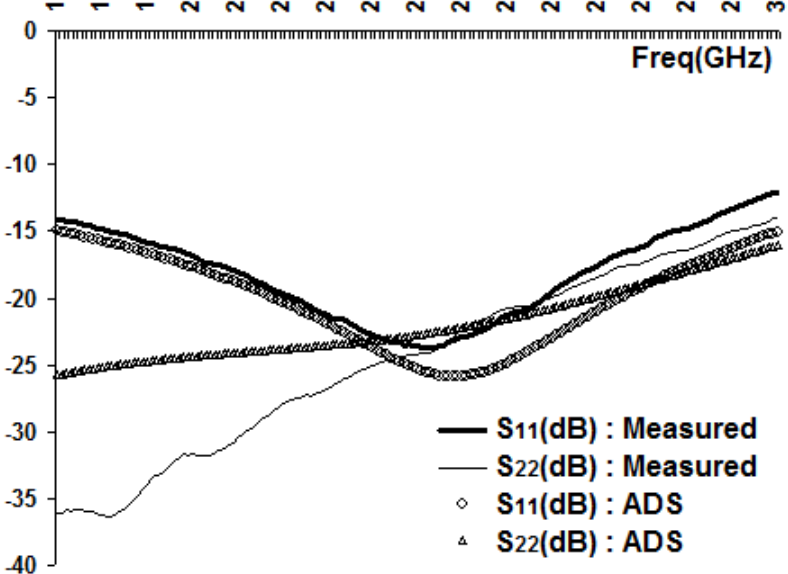

Fig. 10. Variation of the reflection parameters $S_{11}$ and $S_{22}$ as a function of frequency.

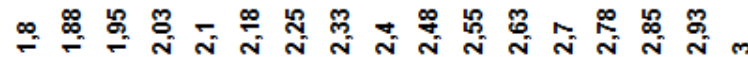

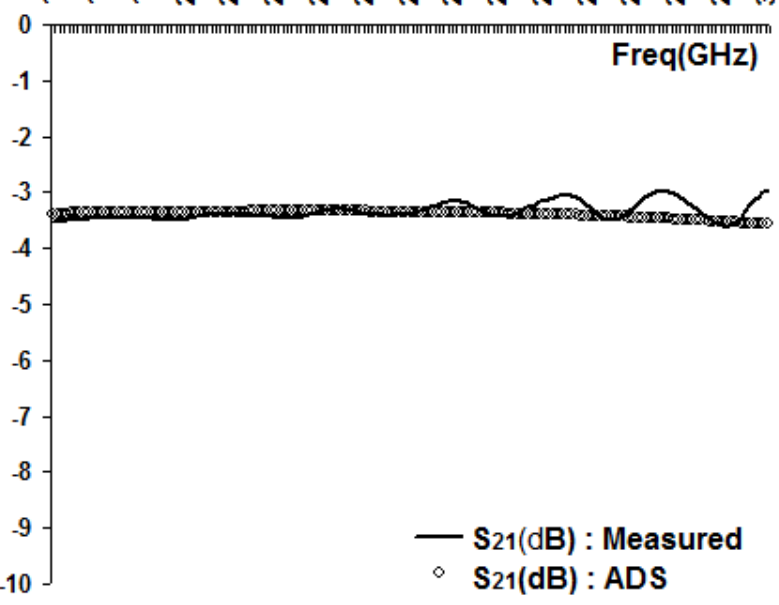

Fig. 11. Variation of transmission parameters $S_{21}$ as a function of frequency. 


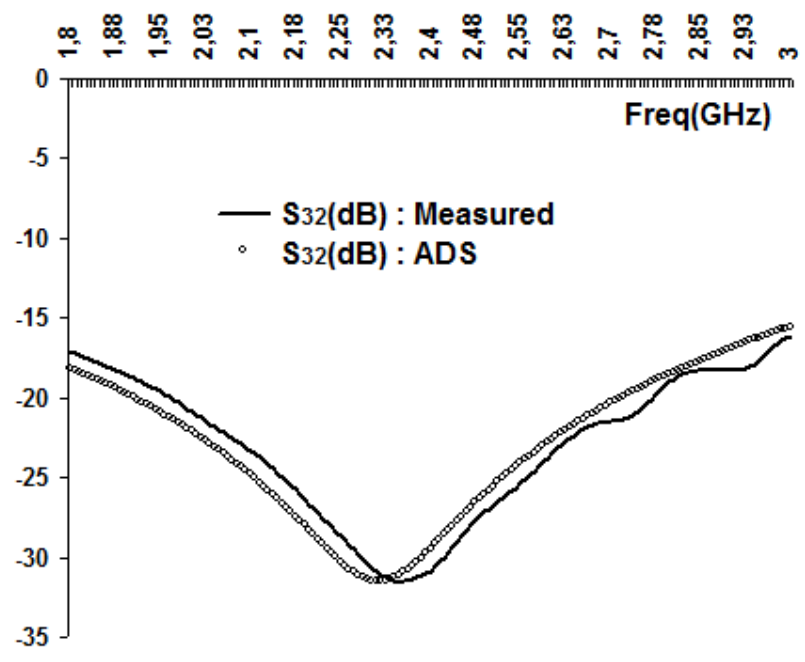

Fig. 12. Variation of transmission parameters $S_{32}$ as a function of frequency.

Figure 11 shows well for $f=2.4 \mathrm{GHz}, S_{21}=S_{31}=$ $-3.38 \mathrm{~dB}$. This means that the energy applied at port 1 will be divided into two equal parts on ports 2 and 3 . The output channels are symmetrical.

Figure 12 shows for $f=2.4 \mathrm{GHz}, S_{23}=S_{32}=-31 \mathrm{~dB}$. This means that ports 2 and 3 are well insulated from one another. Since the sensitive circuits of the receiver cannot support the power signals generated by the transmitter. This isolation is necessary for the separation of the two ports (Tx) and (Rx) of the duplexer.

\section{Experimental Characterization of the Realized Duplexer}

In this last part of this work, we are interested in the integration of all the elements of the duplexer (the two RF amplifiers and the Wilkinson divider) on the same PCB. All the results presented were found by measurements on a vector network analyzer Agilent Technologies E5062A and using the Advanced Design System (ADS) software. In the different simulations carried out, the parameters $S_{i j}$ of the transistor BFR91 (obtained by measurements using a VNA) are introduced into the overall calculation, via a file whose extension is "s $2 p$ ".

Figure 14 shows for $f=2.4 \mathrm{GHz}, S_{11}=-12.2 \mathrm{~dB}$, $S_{22}=-17.3 \mathrm{~dB}, S_{33}=-11.5 \mathrm{~dB}$. This implies that the three ports of the duplexer are well-adapted and the reflections at these ports are low.

The found values of the different isolations observed in Fig. $15\left(S_{12}=-53 \mathrm{~dB}, S_{21}=-22 \mathrm{~dB}, S_{13}=-16 \mathrm{~dB}\right.$ and $S_{32}=-18 \mathrm{~dB}$ at $f=2.4 \mathrm{GHz}$, ) are considered satisfactory for this application (low power).

Indeed, for an input power of $300 \mathrm{~mW}$ (Tx), the return signal (received by the antenna, assumed to be very small in front of the transmission signal) could not disturb the transmission $\left(S_{13}=-16 \mathrm{~dB}\right)$.

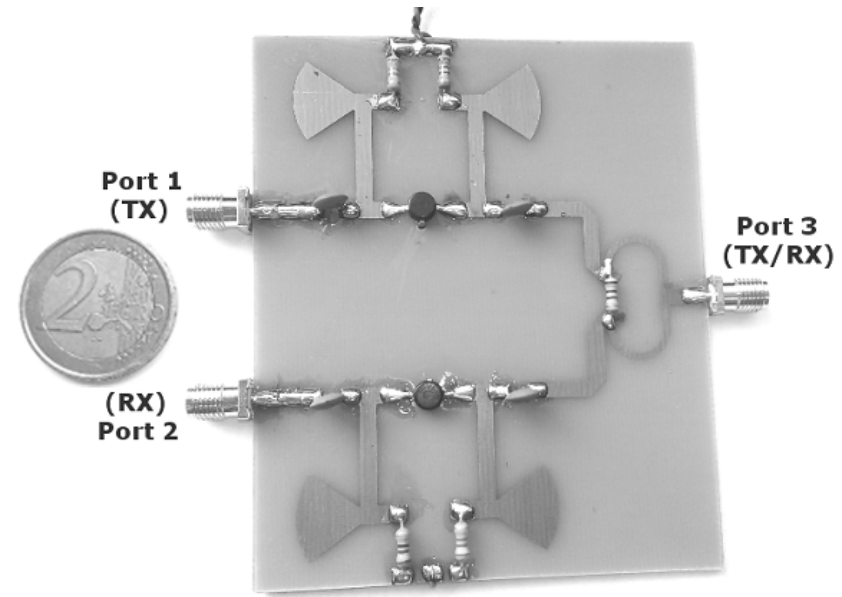

Fig. 13. The realized duplexer.

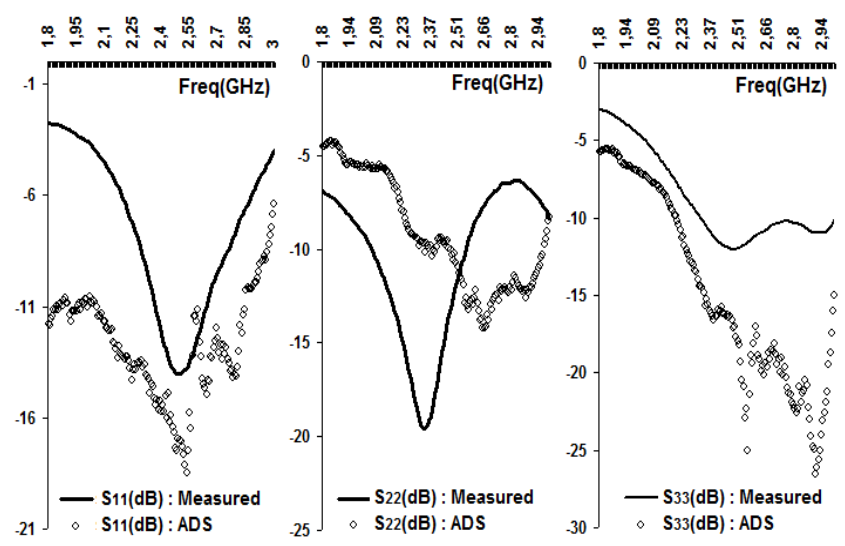

Fig. 14. Variation of the reflection parameters $S_{11}, S_{22}$ and $S_{33}$ as a function of the frequency.

Similarly the signal reflected by the receiver $(\mathrm{Rx})$, which is characterized by low power, for isolation $\left(S_{32}=\right.$ $-18 \mathrm{~dB})$, does not affect the signal received by the antenna.

These results show that the isolation between the transmit (Tx) and receive $(\mathrm{Rx})$ circuits is considered satisfactory for low power applications $\left(S_{21}=-22 \mathrm{~dB}\right)$.

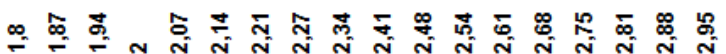

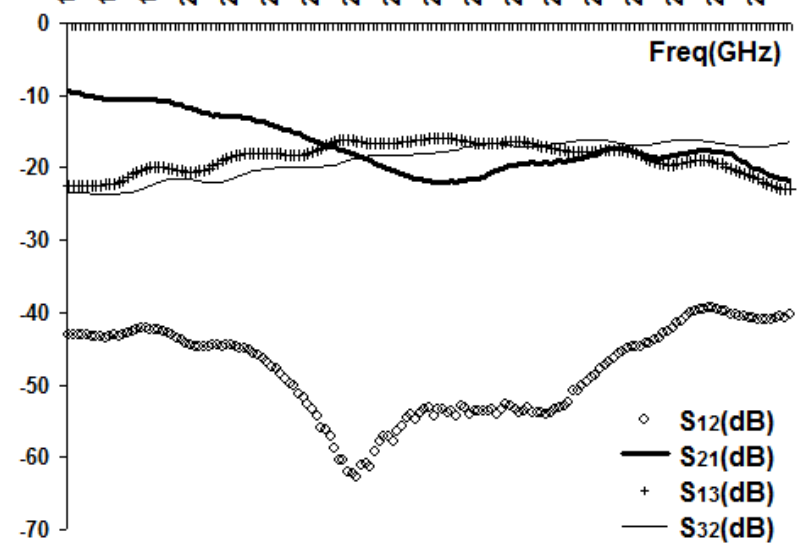

Fig. 15. Variation of transmission parameters $S_{12}, S_{21}, S_{13}$ and $S_{32}$ as a function of the frequency. 


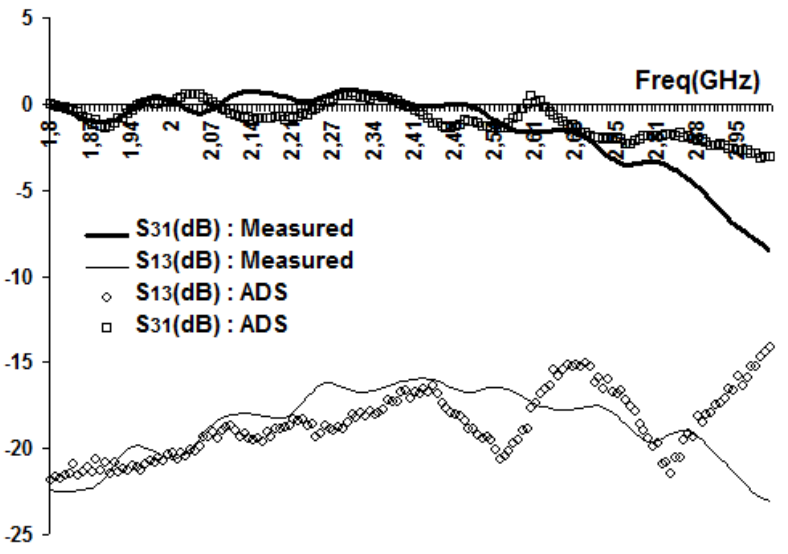

Fig. 16. Variation of transmission parameters $S_{13}$ and $S_{31}$ as a function of frequency.

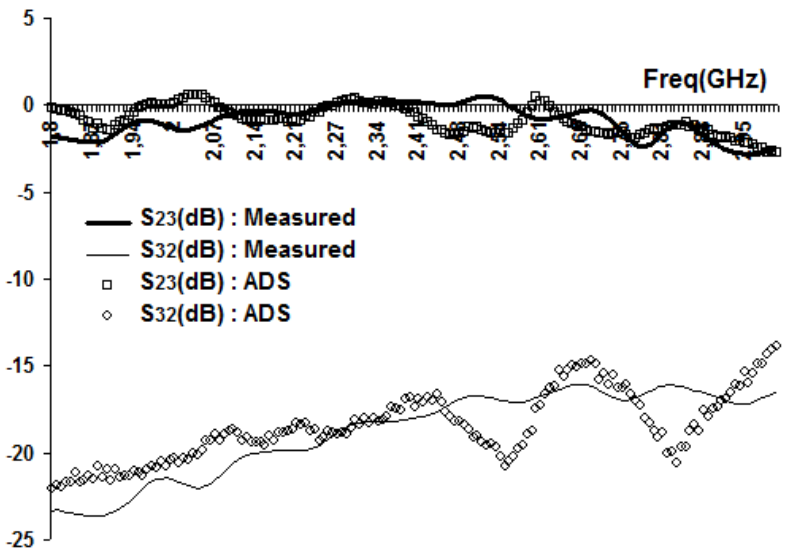

Fig. 17. Variation of transmission parameters $S_{32}$ and $S_{23}$ as a function of frequency.

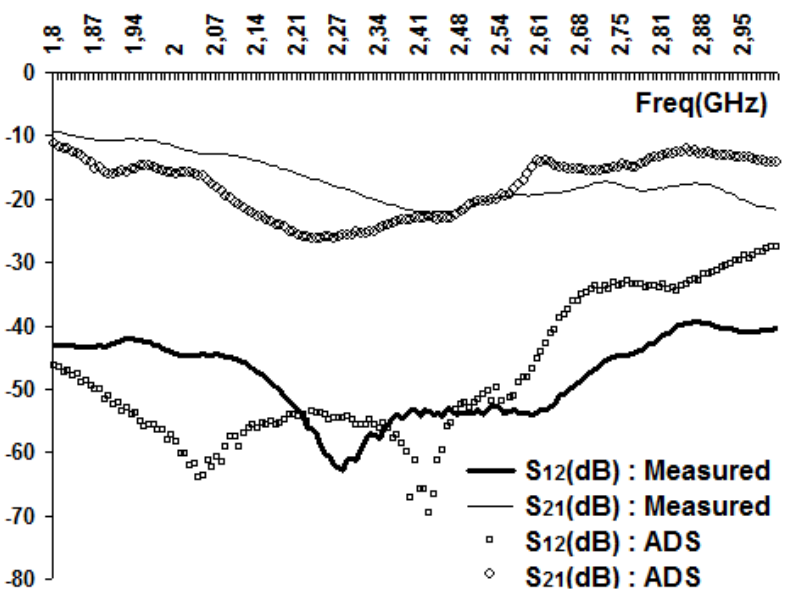

Fig. 18. Variation of transmission parameters $S_{21}$ and $S_{12}$ as a function of frequency.

The isolation between the transmitter and receiver has been assured by the power divider that has an isolation of $31 \mathrm{~dB}$ (see Fig. 12).

Figure 16 shows that for $f=2.4 \mathrm{GHz}, S_{13}=-16 \mathrm{~dB}$ and $S_{31}=-0.047 \mathrm{~dB}$, this implies that the transmission is only one-way, from port 1 to port 3 (from the transmitter to the antenna). This was ensured by the transistor BFR91 which is a unidirectional component placed in the circuit Tx.
Figure 17 shows that for $f=2.4 \mathrm{GHz}, S_{32}=-18 \mathrm{~dB}$ and $S_{23}=-0.2 \mathrm{~dB}$, this implies that the transmission is only one-way, from port 3 to port 2 (from the antenna to the receiver). This was ensured by the transistor BFR91 which is a unidirectional component placed in the circuit $\mathrm{Rx}$.

The use of two BFR91 transistors in cascades, instead of just one, makes it possible to double the values of the parameters $S_{13}$ and $S_{32}$.

Figure 18 shows well for $f=2.4 \mathrm{GHz}, S_{21}=-22 \mathrm{~dB}$ and $S_{12}=-53 \mathrm{~dB}$, which means that ports 1 and 2 are isolated. This prevents the transmitter from transmitting power signals to the receiver which contains very sensitive circuits. This isolation was ensured by Wilkinson's power divider.

The studied duplexer has been experimentally validated. Insertion losses, measured at $2.4 \mathrm{GHz}$ are negligible $\left(S_{31}=-0.047 \mathrm{~dB}\right.$ between the transmitter and the antenna, and $S_{23}=-0.2 \mathrm{~dB}$ between the antenna and the receiver). Due to an insulation that is considered satisfactory, for low power applications. The simulated and measured results show a good agreement.

The noise observed in the various measurement curves (Fig. 7, Fig. 11,...) essentially in high frequency, is due, to our opinion, to the different resonance phenomena that occur in the circuits made (the discontinuities produced by the welds as well as those between the supply lines and the connectors, the capacitors, the transistors, ...) which can have a not negligible effect on the results obtained. These phenomena are often difficult to take into account in simulations.

\section{Conclusion}

In this article, we have concentrated our efforts on the design and realization of a duplexer in planar technology. It integrates available and inexpensive active components. This duplexer works in the microwave band. It is easy to build and integrate into systems such as radar and wireless communications systems. The results we obtained during the study of this device, confirm that all its inputs are adapted and that the isolation between the circuits of emission (Tx) and of reception ( $\mathrm{Rx})$ is considered satisfactory for low power applications. The incoming energy through the Tx port will be directed only to the antenna and the energy delivered by the antenna will be directed to the receiver. Insertion losses are negligible. The power that the duplexer can dissipate as well as its sensitivity are determined by the RF transistors used.

\section{Acknowledgments}

This work has been supported and funded by the Sys'Com Research Laboratory, National Engineering School of Tunis (ENIT) and University Tunis El Manar, Tunis, Tunisia. 


\section{References}

[1] HICKMAN, I. Analog Circuits Cookbook. 2nd ed. Newnes, 1999. ISBN: 0750642343

[2] HAGEN, J. B. Radio-Frequency Electronics Circuits and Applications. 2nd ed. Cambridge University Press, 2009. ISBN: 978-0-521-88974-2

[3] DANIELS, D. J. Ground Penetrating Radar. 2nd ed. London (United Kingdom): Institution of Electrical Engineers, 2003. ISBN: 0863413609

[4] BARTON, D. K., LEONOV, S. A. Radar Technology Encyclopedia. Norwood: Artech House, 1998. ISBN: 0-89006893-3

[5] http://www.radartutorial.eu/06.antennas/Duplexeur\%20lat\%C3\%A 9ral.fr.html

[6] ANNAN, A. P. Ground Penetrating Radar Principles, Procedures \& Applications. Sensors \& Software Inc., 2003.

[7] BRISTOW, C. S., JOL, H. M. (Eds.) Ground Penetrating Radar in Sediments. Geological Society of London, 2003. ISBN: 1-86239$131-9$

[8] GONZALEZ, G. Microwave Transistor Amplifiers, Analysis and Design. 2nd ed. Upper Saddle River, NJ: Prentice-Hall, Inc. 1997. ISBN: 0-13-254335-4

[9] WADELL, B. C. Transmission Line Design Handbook. Artech House, 1991. ISBN: 0-89006-436-9

[10] HONG, J. S., LANCASTER, M. J. Microstrip Filters for RF/Microwave Applications. John Wiley, 2001. ISBN: 0-47122161-9

[11] FOOKS, E. H., ZAKAREVICIUS, R. A. Microwave Engineering Using Microstrip Circuits. Prentice Hall, 1990. ISBN: 0136916503

[12] WONG, K. L. Compact and Broadband Microstrip Antennas. John Wiley, 2002. ISBN: 0-471-41717-3
[13] WILKINSON, E. J. An N-way hybrid power divider. IRE Transactions on Microwave Theory and Techniques, 1960, vol. 8, no. 1, p. 116-118. DOI: 10.1109/TMTT.1960.1124668

[14] HORST, S., BAIRAVASUBRAMANIAN, R., TENTZERIS, M. M., et al. Modified Wilkinson power dividers for millimeter-wave integrated circuits. IEEE Transactions on Microwave Theory and Techniques, 2007, vol. 55, no. 11, p. 2439-2445. DOI: 10.1109/TMTT.2007.908672

\section{About the Authors ...}

Fethi MEJRI was born in Tunisia. Assistant professor at the Faculty of Science of Bizerte (FSB), Tunisia. He received the diploma of engineer in Electronics from the National Engineering School of Tunis (ENIT), in 1993, and the Diploma of Advanced Study in Analysis of Digital Systems and Processing from the ENIT, in 1998. The doctoral degree in telecommunications from the ENIT, in 2006. His research activities include electromagnetic modeling, design and realization of microwave circuits.

Taoufik AGUILI was born in Tunisia. He received the diploma of DEA in Integrated Electronics INSA Lyon, in 1987. The Diploma doctorate in Integrated Electronics UCBL Lyon, in 1990. Appointed Head of "Communications Systems" at the ENIT, in 1998. Diploma of doctorate of state in electrical engineering of the National School of Engineers of Tunis, in 1999. Appointed professor in Telecommunications at the National School of Engineers of Tunis, in 2004. Appointed Director of Communications Systems Laboratory at ENIT, in 2011. His research activities include electromagnetic microwave circuits modeling and analysis of scattering and propagation phenomena in free space. 